\title{
HOPF BIFURCATION OF AN HIV-1 VIRUS MODEL WITH TWO DELAYS AND LOGISTIC GROWTH*
}

\author{
Caixia Sun, Lele Li And Jianwen Jia**
}

\begin{abstract}
The paper establish and investigate an HIV-1 virus model with logistic growth, which also has intracellular delay and humoral immunity delay. The local stability of feasible equilibria are established by analyzing the characteristic equations. The globally stability of infection-free equilibrium and immunity-inactivated equilibrium are studied using the Lyapunov functional and LaSalles invariance principle. Besides, we prove that Hopf bifurcation will occur when the humoral immune delay pass through the critical value. And the stability of the positive equilibrium and Hopf bifurcations are investigated by using the normal form theory and the center manifold theorem. Finally, we confirm the theoretical results by numerical simulations.
\end{abstract}

Mathematics Subject Classification. D25, 34C23, 37B25, 34D23.

Received December 28, 2018. Accepted September 8, 2019.

\section{INTRODUCTION}

For the past few years, HIV-1 infection model has been widely studied (see [4-6, 11, 13, 20]). HIV-1 has two primary infection modes, one is the classical cell-free transmission [6], and the other is direct cell-to-cell transfer of viral particles [5]. A great amount of the studies often only consider one aspect. For instance, Song and Wang [21] and Wang [28] established a viral infection model with lytic and nonlytic immune responses. Li and Wang [15] discussed an HIV infection model which incorporated directly cell-to-cell transmission. In Lai and Zou [13, 14], the author considered the effect of cell-to-cell transfer of HIV-1 on the virus dynamics. Wang et al. [26] studied age-structured viral infection models with cell-to-cell transmission. In [8], Elaiw and Alshamrani analyzed two nonlinear viral infection models with humoral immune response. Murase et al. [18] and Wang and Zou [29] investigated a basic HIV-1 virus model with humoral immunity. In [15], Feng Li and JingLiang Wang considered an HIV-1 infection model with logistic growth. Hu and co-workers [12] studied the dynamics of a delayed viral infection model with logistic growth and immune impairment. Furthermore, time delays (see $[7,22,23]$ ) have been applied to epidemic models for the sake of having a better understanding of more complicated phenomena for describing several aspects of infectious disease dynamics. On the influence of time delay on model dynamics, scholars mainly discussed the influence of time delay on the stability of system equilibrium point and the existence of Hopf bifurcation [1, 19]. In [17], authors considered an HIV-1 virus model to describe virus-to-cell, cell-to-cell transmissions, intracellular delay and humoral immunity. The model is as

${ }^{*}$ This work is supported by Natural Science Foundation of Shanxi province (201801D121011).

Keywords and phrases: HIV-1 virus model, delay, stability, Hopf bifurcation, logistic growth.

School of Mathematics and Computer Science, Shanxi Normal University, Linfen 041004, Shanxi, PR China.

** Corresponding author: Jiajw. 2008@163.com 
follows:

$$
\left\{\begin{array}{l}
\frac{\mathrm{d} x(t)}{\mathrm{d} t}=\Lambda-d x(t)-\beta_{1} x(t) v(t)-\beta_{2} x(t) y(t), \\
\frac{\mathrm{d} y(t)}{\mathrm{d} t}=\beta_{1} e^{-m \tau_{1}} x(t-\tau) v(t-\tau)+\beta_{2} e^{-m \tau_{1}} x(t-\tau) y(t-\tau)-a y(t), \\
\frac{\mathrm{d} v(t)}{\mathrm{d} t}=k y(t)-u v(t)-p v(t) z(t), \\
\frac{\mathrm{d} z(t)}{\mathrm{d} t}=c v(t) z(t)-b z(t),
\end{array}\right.
$$

denote by $x(t), v(t), v(t)$ and $z(t)$, the uninfected cells (susceptible cells), infected cells, virus and B cells. Uninfected cells (susceptible cells) are produced at rate $\Lambda$ and died at rate $d, \beta_{1}$ and $\beta_{2}$ are infection rates of virus-to-cell transmission and cell-to-cell transmission, respectively; the delay $\tau$ represents the time between viral entry into a cell and the production of new free virus or the time between infected cells spreading virus into uninfected cells and the production of new free virus; $m$ is assumed to be a constant death rate for infected but not yet virus-producing cells. Thus, the feasibility of surviving the time period from $t-\tau$ to $t$ is $e^{-m \tau}$; $a, u$ and $b$ are death rates of infected cells, virus and B cells, respectively; $k$ denotes the number of free virus particles produced by per infected cell. $p v(t) z(t)$ is used to describe the virus killed by B cells and $c v(t) z(t)$ is is used to describe the new $\mathrm{B}$ cells produced by antigenic stimulation.

However, time delays cannot be ignored in models for immune response as shown in [3, 25, 27]. Besides, we can see that the logistic growth is considered in $[14,15]$. Therefore, we propose the following delay and logistic growth model motivated by the works of [17] in the present study,

$$
\left\{\begin{array}{l}
\frac{\mathrm{d} x(t)}{\mathrm{d} t}=r x(t)\left(1-\frac{x(t)}{x_{M}}\right)-\beta_{1} x(t) v(t)-\beta_{2} x(t) y(t), \\
\frac{\mathrm{d} y(t)}{\mathrm{d} t}=\beta_{1} \alpha x\left(t-\tau_{1}\right) v\left(t-\tau_{1}\right)+\beta_{2} \alpha x\left(t-\tau_{1}\right) y\left(t-\tau_{1}\right)-a y(t), \\
\frac{\mathrm{d} v(t)}{\mathrm{d} t}=k y(t)-u v(t)-p v(t) z(t), \\
\frac{\mathrm{d} z(t)}{\mathrm{d} t}=c v\left(t-\tau_{2}\right) z\left(t-\tau_{2}\right)-b z(t),
\end{array}\right.
$$

where the state variables $x, y, v, z$ and all parameters have same biological meanings as in the model (1.0). $\tau_{2}$ represents the time that HIV-1 virus stimulation needs for generating B cells [3]. Here, target cells grow at a rate $r$ and this growth is limited by a carrying target cells $x_{M}$ and the probability of surviving the time period from $t-\tau_{1}$ to $t$ is $\alpha$. All parameters are assumed to be positive.

Let $\tau=\max \left\{\tau_{1}, \tau_{2}\right\}$. The initial conditions for system (1.2) take the form

$$
\begin{aligned}
& x(\theta)=\phi_{1}(\theta), y(\theta)=\phi_{2}(\theta), v(\theta)=\phi_{3}(\theta), z(\theta)=\phi_{4}(\theta), \\
& \phi_{i}(\theta) \geq 0, \theta \in[-\tau, 0), \phi_{i}(0)>0, i=1,2,3,4,
\end{aligned}
$$

where $\phi_{i}(\theta) \in C\left([-\tau, 0], R_{+0}^{4}\right), i=1,2,3,4$, is the Banach space of continuous functions mapping the interval $[-\tau, 0]$ into $R_{+0}^{4}$, where $R_{+0}^{4}=\left\{\left(x_{1}, x_{2}, x_{3}, x_{4}\right): x_{i} \geq 0, i=1,2,3,4\right\}$.

It can be proved that system (1.2) has an unique solution $(x(t), y(t), v(t), z(t))$ that satisfying the initial condition (1.3) by the fundamental theory of functional differential equations [9]. It is easy to show that all solutions of system (1.2) with initial condition (1.3) are defined on $[0,+\infty)$ and remain positive for all $t \geq 0$.

The rest of the paper is organized as follows. In Section 2, we investigate and verify the existence of feasible equilibria and the stability of nonnegative equilibria. In Section 3, we determine the stability of positive equilibrium and the existence of Hopf bifurcation. In Section 4, the explicit formulate for determining the direction of Hopf bifurcation and the stability of bifurcating periodic solutions are proved by using the normal form theory and the center manifold theorem. In order to verify our theoretical prediction, some numerical simulations are also included in Section 5. Finally, in Section 6, we give some remarks on the biological interpretation of our results, and some further extensions of the model one can make. 


\section{Existence of EQUiLibria AND THE StABILITY OF NONNEGATIVE EQUILIBRIA}

\subsection{Feasible equilibria}

Considering the existence of the three equilibria, we get the following results.

Denote $\Re_{0}=\frac{\left(\alpha \beta_{1} k^{+} \alpha \beta_{2} u\right)}{a u} x_{M}$, which is called immune-inactivated reproduction rate of system (1.2), it represents the number of newly infected cells produced by one infected cell during its lifespan.

$$
\Re_{1}=\frac{\Re_{0}}{1+\frac{a b u}{c k x_{M}\left(\frac{2 x_{1}}{x_{M}}-r\right) \alpha} \Re_{0}}=\frac{\beta_{1} k+\beta_{2} u}{c k\left(\frac{2 r x_{1}}{x_{M}}-r+\frac{a b u}{x_{M}} \Re_{0}\right)}\left(c v_{1}-b\right)+1,
$$

which is called immune-activated reproduction rate.

Clearly, system (1.2) always has an infection-free equilibrium $E_{0}\left(x_{M}, 0,0,0\right)$.

If $\Re_{0}>1$, there exists an immunity-inactivated equilibrium $E_{1}\left(x_{1}, y_{1}, v_{1}, 0\right)$, and $E_{0}$, where

$$
x_{1}=\frac{a u}{\alpha \beta_{1} k+\alpha \beta_{2} u}, \quad y_{1}=r \Re_{0}\left(\Re_{0}-1\right) \frac{u}{\beta_{1} k+\beta_{2} u}, \quad v_{1}=r \Re_{0}\left(\Re_{0}-1\right) \frac{k}{\beta_{1} k+\beta_{2} u} .
$$

If $\Re_{1}>1$, there exists an immunity-activated equilibrium $E_{2}\left(x_{2}, y_{2}, v_{2}, z_{2}\right), E_{0}$ and $E_{1}$, where

$$
y_{2}=\frac{p b z_{2}+u b}{c k}, \quad v_{2}=\frac{b}{c}, \quad x_{2}=\frac{a p b z_{2}+a u b}{\alpha \beta_{1} b k+\alpha \beta_{2}\left(p b z_{2}+u b\right)},
$$

and $z_{2}$ is the unique positive real root of the following quadratic equation:

$$
\begin{gathered}
p^{2} b \alpha \beta_{2} z^{2}(t)+\left(\alpha r c k p+\alpha \beta_{1} p b k x_{M}-\alpha r p c k x_{M}+\alpha \beta_{2} u b p x_{M}+\alpha \beta_{2} p b u x_{M}\right) z(t) \\
+\left(\alpha \beta_{2} u^{2} b x_{M}+\alpha \beta_{1} b k u x_{M}+\alpha c d k u x_{M}\right)\left(1-\Re_{1}\right)=0 .
\end{gathered}
$$

\subsection{The stability of the virus-free equilibrium}

Theorem 2.1. For any $\tau_{1}, \tau_{2}$, if $\Re_{0}<1$, the infection-free equilibrium $E_{0}$ of system (1.2) is locally asymptotically stable; if $\Re_{0}>1, E_{0}$ is unstable.

Proof. The characteristic equation of system (1.2) at $E_{0}$ is

$$
(\lambda+r)(\lambda+b)\left[(\lambda+a)(\lambda+u)-e^{-\lambda \tau_{1}} x_{M}\left(\alpha \beta_{2} \lambda+k \alpha \beta_{1}+u \alpha \beta_{2}\right)\right]=0 .
$$

It's clear that equation (2.1) have negative real parts roots $\lambda=-r, \lambda=-b$ and other roots are determined by following equation

$$
G(\lambda) \equiv(\lambda+a)(\lambda+u)-e^{-\lambda \tau_{1}} x_{M}\left(\alpha \beta_{2} \lambda+k \alpha \beta_{1}+u \alpha \beta_{2}\right)=0 .
$$

Denote $\Re_{0}=\Re_{01}+\Re_{02}$, where $\Re_{01}=\frac{\alpha \beta_{1} k x_{M}}{a u}, \Re_{02}=\frac{\alpha \beta_{2} x_{M}}{a}$. Direct calculation shows that

$$
\left(\frac{\lambda}{u}+1\right)\left(\frac{\lambda}{a}+1\right)=e^{-\lambda \tau_{1}}\left(\frac{\lambda}{u} \Re_{02}+\Re_{0}\right) .
$$


Now, we claim that all roots of (2.3) has negative real parts. Otherwise, there exists a root $\lambda_{1}=x_{1}+i y_{1}$ with $x_{1} \geq 0$. In this case, if $\Re_{0}<1$, it is easy to see that

$$
\left|\frac{\lambda_{1}}{u}+1\right|>\left|\frac{\lambda_{1}}{u} \Re_{02}+\Re_{0}\right|, \quad\left|\frac{\lambda_{1}}{a}+1\right|>e^{-x_{1} \tau_{1}} .
$$

It follows that

$$
\left|\left(\frac{\lambda_{1}}{u}+1\right)\left(\frac{\lambda_{1}}{a}+1\right)\right|>\left|e^{-\lambda \tau_{1}}\left(\frac{\lambda_{1}}{u} \Re_{02}+\Re_{0}\right)\right|,
$$

which contradicts to (2.3). Therefore, if $\Re_{0}<1$, all roots of (2.1) have negative real parts and $E_{0}$ is locally asymptotically stable.

If $\Re_{0}>1, G(0)=a u\left(1-\Re_{0}\right)<0$ and $G(\lambda) \rightarrow+\infty$ as $\lambda \rightarrow+\infty$. So equation (2.2) has a positive real root, i.e. equation (2.1) has a positive real root, Hence, $E_{0}$ is unstable.

Theorem 2.2. For any $\tau_{1}, \tau_{2}$, the virus-free equilibrium $E_{0}$ is globally asymptotically stable if $\Re_{0}<1$.

Proof. Define a Lyapunov functional as follows

$$
\begin{aligned}
V_{1}(t)= & x(t)-x_{0}-x_{0} \ln \frac{x(t)}{x_{0}}+c_{1} y(t)+c_{2} v(t)+c_{3} z(t) \\
& +\int_{t-\tau_{1}}^{t}\left(\beta_{1} x(s) v(s)+\beta_{2} x(s) y(s)\right) \mathrm{d} s+c_{2} \int_{t-\tau_{2}}^{t} p v(s) z(s) \mathrm{d} s,
\end{aligned}
$$

where $x_{0}=x_{M}$ and $c_{1}, c_{2}$ and $c_{3}$ will be determined later.

Calculating the derivative of $V_{1}(t)$ along the solutions of system (1.2), we obtain that

$$
\begin{aligned}
\frac{\mathrm{d} V_{1}(t)}{\mathrm{d} t}= & \left(1-\frac{x_{0}}{x(t)}\right) \frac{\mathrm{d} x(t)}{\mathrm{d} t}+c_{1} \frac{\mathrm{d} y(t)}{\mathrm{d} t}+c_{2} \frac{\mathrm{d} v(t)}{\mathrm{d} t}+c_{3} \frac{\mathrm{d} z(t)}{\mathrm{d} t} \\
& +\beta_{1} x(t) v(t)+\beta_{2} x(t) y(t)-\beta_{1} x\left(t-\tau_{1}\right) v\left(t-\tau_{1}\right)-\beta_{2} x\left(t-\tau_{1}\right) y\left(t-\tau_{1}\right) \\
& +c_{2} p v(t) z(t)-c_{2} p v\left(t-\tau_{2}\right) z\left(t-\tau_{2}\right)
\end{aligned}
$$

Direct calculation shows that

$$
\begin{aligned}
\frac{\mathrm{d} V_{1}(t)}{\mathrm{d} t}= & -r \frac{\left(x(t)-x_{0}\right)^{2}}{x_{M}}+\left(c_{2} k+\beta_{2} x_{0}-c_{1} a\right) y(t)-c_{3} b z(t) \\
& +\left(c_{1} \alpha-1\right)\left[\beta_{1} x\left(t-\tau_{1}\right) v\left(t-\tau_{1}\right)+\beta_{2} x\left(t-\tau_{1}\right) y\left(t-\tau_{1}\right)\right] \\
& +\left(\beta_{1} x_{0}-c_{2} u\right) v(t)+\left(c_{3} c-p c_{2}\right) v\left(t-\tau_{2}\right) z\left(t-\tau_{2}\right) .
\end{aligned}
$$

Let

$$
c_{1}=\frac{1}{\alpha}, \quad c_{2}=\frac{\beta_{1}}{u} x_{M}, \quad c_{3}=\frac{\beta_{1} p}{c u} x_{M} .
$$

Thus,

$$
\frac{\mathrm{d} V_{1}(t)}{\mathrm{d} t}=-r \frac{\left(x(t)-x_{0}\right)^{2}}{x_{M}}+\alpha a\left(\Re_{0}-1\right) y(t)-\frac{\beta_{1} p b}{c u} x_{M} z(t) .
$$


It follows from (2.4) that $\frac{\mathrm{d} V_{1}(t)}{\mathrm{d} t} \leq 0$ with equality holding if and only if $x=x_{0}, y=v=z=0$. It can be certified that $M_{0}=\left\{E_{0}\right\} \subset \Omega$ is the largest invariant subset of $\left\{(x(t), y(t), v(t), z(t)): \frac{\mathrm{d} V_{1}}{\mathrm{~d} t}=0\right\}$. According to Theorem 2.1, $E_{0}$ is locally asymptotically stable. From LaSalles invariance principle, we obtain that $E_{0}$ is globally asymptotically stable.

\subsection{The stability of the immunity-inactivated equilibrium}

Theorem 2.3. For any $\tau_{1}, \tau_{2}$, the immunity-inactivated equilibrium $E_{1}$ of system (1.2) is locally asymptotically stable if $\Re_{1}<1<\Re_{0}$.

Proof. The characteristic equation of system (1.2) at $E_{1}$ is

$$
\left(\lambda+b-c v_{1} e^{-\lambda \tau_{2}}\right)\left[(\lambda+a)(\lambda+u)\left(\lambda+r-\frac{2 r x_{1}}{x_{M}}+\beta_{1} v_{1}+\beta_{2} y_{1}\right)-e^{-\lambda \tau_{1}} x_{1}\left(\lambda+r-\frac{2 r x_{1}}{x_{M}}\right)\left(\alpha \beta_{2} \lambda+\alpha \beta_{1} k+\alpha \beta_{2} u\right)\right]=0 .
$$

It is clear that $\left(\lambda+b-c v_{1} e^{-\lambda \tau_{2}}\right)=0$ has a negative real root when $\Re_{1}<1$, and other roots are determined by the following equation:

$$
(\lambda+a)(\lambda+u)\left(\lambda+r-\frac{2 r x_{1}}{x_{M}}+\beta_{1} v_{1}+\beta_{2} y_{1}\right)-e^{-\lambda \tau_{1}} x_{1}\left(\lambda+r-\frac{2 r x_{1}}{x_{M}}\right)\left(\alpha \beta_{2} \lambda+\alpha \beta_{1} k+\alpha \beta_{2} u\right)=0 .
$$

Direct calculation shows that

$$
\left(\frac{\lambda}{a}+1\right)\left(\frac{\lambda}{u}+1\right)\left(\frac{\lambda}{r-\frac{2 r x_{1}}{x_{M}}}+\Re_{0}\right)=e^{-\lambda \tau_{1}}\left(\frac{\lambda}{r-\frac{2 r x_{1}}{x_{M}}}+1\right)\left(\frac{\lambda}{u} \frac{\Re_{02}}{\Re_{0}}+1\right) .
$$

Now, we claim that all roots of (2.7) has negative real parts. Otherwise, there exists a root $\lambda_{2}=x_{2}+i y_{2}$ with $x_{2} \geq 0$. In this case, if $\Re_{0}>1$, it is easy to see that

$$
\left|\frac{\lambda_{2}}{a}+1\right|>e^{-x_{1} \tau_{1}},\left|\frac{\lambda_{2}}{r-\frac{2 r x_{1}}{x_{M}}}+\Re_{0}\right|>\left|\frac{\lambda_{2}}{r-\frac{2 r x_{1}}{x_{M}}}+1\right|, \quad\left|\frac{\lambda_{2}}{u}+1\right|>\left|\frac{\lambda_{2}}{u} \frac{\Re_{02}}{\Re_{0}}+1\right| .
$$

It follows that

$$
\left|\left(\frac{\lambda_{2}}{a}+1\right)\left(\frac{\lambda_{2}}{u}+1\right)\left(\frac{\lambda_{2}}{r-\frac{2 r x_{1}}{x_{M}}}+\Re_{0}\right)\right|>\left|e^{-\lambda_{2} \tau_{1}}\left(\frac{\lambda_{2}}{r-\frac{2 r x_{1}}{x_{M}}}+1\right)\left(\frac{\lambda_{2}}{u} \frac{\Re_{02}}{\Re_{0}}+1\right)\right| .
$$

which leads to a contradiction to (2.7). Thus, all roots of equation (2.6) have negative real parts, and $E_{1}$ is locally asymptotically stable.

Theorem 2.4. For any $\tau_{1}, \tau_{2}$, the immunity-inactivated equilibrium $E_{1}$ is globally asymptotically stable if $\Re_{1}<1<\Re_{0}$.

Proof. Consider the following Lyapunov functional

$$
\begin{aligned}
V_{2}(t)= & x(t)-x_{1}-x_{1} \ln \frac{x(t)}{x_{1}}+k_{1}\left(y(t)-y_{1}-y_{1} \ln \frac{y(t)}{y_{1}}\right)+k_{2}\left(v(t)-v_{1}-v_{1} \ln \frac{v(t)}{v_{1}}\right) \\
& +k_{3} z(t)+\beta_{1} x_{1} v_{1} \int_{t-\tau_{1}}^{t}\left[\frac{x(s) v(s)}{x_{1} v_{1}}-1-\ln \frac{x(s) v(s)}{x_{1} v_{1}}\right] \mathrm{d} s \\
& +\beta_{2} x_{1} y_{1} \int_{t-\tau_{1}}^{t}\left[\frac{x(s) y(s)}{x_{1} y_{1}}-1-\ln \frac{x(s) y(s)}{x_{1} y_{1}}\right] \mathrm{d} s+k_{2} \int_{t-\tau_{2}}^{t} p v(s) z(s) \mathrm{d} s,
\end{aligned}
$$


where constants $k_{1}, k_{2}$ and $k_{3}$ will be determined later.

Calculating the derivative of $V_{2}(t)$ along the solutions of system (1.2), we have

$$
\begin{aligned}
\frac{\mathrm{d} V_{2}(t)}{\mathrm{d} t}= & -r \frac{\left(x(t)-x_{1}\right)^{2}}{x_{M}}+\beta_{1} x_{1} v_{1}\left(1+k_{1} \alpha-\frac{x_{1}}{x(t)}-\frac{x\left(t-\tau_{1}\right) v\left(t-\tau_{1}\right) y_{1}}{x_{1} v_{1} y(t)} k_{1} \alpha+\ln \frac{x\left(t-\tau_{1}\right) v\left(t-\tau_{1}\right)}{x(t) v(t)}\right) \\
& +\beta_{2} x_{1} y_{1}\left(1+k_{1} \alpha-\frac{x_{1}}{x(t)}-\frac{x\left(t-\tau_{1}\right) y\left(t-\tau_{1}\right) y_{1}}{x_{1} y_{1} y(t)} k_{1} \alpha+\ln \frac{x\left(t-\tau_{1}\right) y\left(t-\tau_{1}\right)}{x(t) y(t)}\right) \\
& +\left(k_{1} \alpha-1\right)\left(\beta_{1} x\left(t-\tau_{1}\right) v\left(t-\tau_{1}\right)+\beta_{2} x\left(t-\tau_{1}\right) y\left(t-\tau_{1}\right)\right) \\
& +\left(\beta_{2} x_{1}+k_{2} k-k_{1} \frac{\beta_{1} \alpha x_{1} v_{1}+\beta_{2} \alpha x_{1} y_{1}}{y_{1}}\right) y(t) \\
& +\left(\beta_{1} x_{1}-k_{2} \frac{k y_{1}}{v_{1}}\right) v(t)+\left(k_{2} p v_{1}-k_{3} b\right) z(t) \\
& -k_{2} k v_{1} \frac{y(t)}{v(t)}+k_{2} k y_{1}+\left(k_{3} c-k_{2} p\right) v\left(t-\tau_{2}\right) z\left(t-\tau_{2}\right)
\end{aligned}
$$

Let $k_{1}=\frac{1}{\alpha}, \quad k_{2}=\frac{\beta_{1} x_{1}}{u}, \quad k_{3}=\frac{\beta_{1} p x_{1}}{c u}$. It follows that

$$
\begin{aligned}
\frac{\mathrm{d} V_{2}(t)}{\mathrm{d} t}= & -r \frac{\left(x(t)-x_{1}\right)^{2}}{x_{M}}+\frac{\beta_{1} p x_{1}}{c u}\left(c v_{1}-b\right) z(t)-\beta_{1} x_{1} v_{1}\left(\frac{x_{1}}{x(t)}-1-\ln \frac{x_{1}}{x(t)}\right) \\
& -\beta_{1} x_{1} v_{1}\left(\frac{y(t) v_{1}}{v(t) y_{1}}-1-\ln \frac{y(t) v_{1}}{v(t) y_{1}}\right)-\beta_{1} x_{1} v_{1}\left(\frac{x\left(t-\tau_{1}\right) v\left(t-\tau_{1}\right) y_{1}}{x_{1} v_{1} y(t)}-1-\ln \frac{x\left(t-\tau_{1}\right) v\left(t-\tau_{1}\right) y_{1}}{x_{1} v_{1} y(t)}\right) \\
& -\beta_{2} x_{1} y_{1}\left(\frac{x_{1}}{x(t)}-1-\ln \frac{x_{1}}{x(t)}\right)-\beta_{2} x_{1} y_{1}\left(\frac{x\left(t-\tau_{1}\right) y\left(t-\tau_{1}\right)}{x_{1} y(t)}-1-\ln \frac{x\left(t-\tau_{1}\right) y\left(t-\tau_{1}\right)}{x_{1} y(t)}\right)
\end{aligned}
$$

It follows from (2.8) that $\frac{\mathrm{d} V_{2}(t)}{\mathrm{d} t} \leq 0$ with equality holding if and only if $x=x_{1}, y=y_{1}, v=v_{1}, z=0$. It can be certified that $M_{1}=\left\{E_{1}\right\} \subset \Omega$ is the largest invariant subset of $\left\{(x(t), y(t), v(t), z(t)): \frac{\mathrm{d} V_{2}}{\mathrm{~d} t}=0\right\}$. According to Theorem 2.3, $E_{1}$ is locally asymptotically stable. From LaSalles invariance principle, we obtain that $E_{1}$ is globally asymptotically stable.

\section{The Stability of The immunity-ACTIVATEd Equilibrium And Hopf BIFURCATION}

Theorem 3.1. For any $\tau_{1}$, if $\tau_{2}=0$ and $\Re_{1}>1$ the immunity-activated equilibrium $E_{2}$ of system (1.2) is locally asymptotically stable.

Proof. Noting that $b-c v_{2}=0$, the characteritic equation of system (1.2) at $E_{2}$ is

$$
\begin{aligned}
& \left(\lambda+r-\frac{2 r x_{2}}{x_{M}}+\beta_{1} v_{2}+\beta_{2} y_{2}\right)(\lambda+a)\left[\lambda^{2}+\left(u+p z_{2}\right) \lambda+p z_{2} c v_{2}\right]\left(\beta_{1} v_{2}+\beta_{2} y_{2}\right) \\
& -\left(\lambda+r-\frac{2 r x_{2}}{x_{M}}\right) a e^{-\lambda \tau_{1}}\left[\beta_{2} y_{2}\left(\lambda^{3}+\left(u+p z_{2}\right) \lambda+p z_{2} c v_{2}\right)+k \beta_{1} y_{2} \lambda\right]=0 .
\end{aligned}
$$

Similarly, we claim that all roots of (3.1) has negative real parts. Otherwise, there exists a root $\lambda_{3}=x_{3}+i y_{3}$ with $x_{3} \geq 0$. In this case, if $\Re_{1}>1$, it is easy to see that

$$
\left|\lambda_{3}+r-\frac{2 r x_{2}}{x_{M}}+\beta_{1} v_{2}+\beta_{2} y_{2}\right|>\left|\lambda_{3}+r-\frac{2 r x_{2}}{x_{M}}\right| .
$$


Since

$$
\begin{aligned}
& {\left[\lambda_{3}^{2}+\left(u+p z_{2}\right) \lambda_{3}+p z_{2} c v_{2}\right]\left(\beta_{1} v_{2}+\beta_{2} y_{2}\right)-\left[\beta_{2} y_{2}\left(\lambda_{3}^{2}+\left(u+p z_{2}\right) \lambda_{3}+p z_{2} c v_{2}\right)+k \beta_{1} y_{2} \lambda_{3}\right]} \\
& =\beta_{1} v_{2} \lambda_{3}^{2}+\beta_{1} p c v_{2}^{2} z_{2}
\end{aligned}
$$

We obtain that

$$
\left|\left[\lambda_{3}^{2}+\left(u+p z_{2}\right) \lambda_{3}+p z_{2} c v_{2}\right]\left(\beta_{1} v_{2}+\beta_{2} y_{2}\right)\right|>\left|\beta_{2} y_{2}\left(\lambda_{3}^{2}+\left(u+p z_{2}\right) \lambda_{3}+p z_{2} c v_{2}\right)+k \beta_{1} y_{2} \lambda_{3}\right|
$$

It follows that

$$
\begin{aligned}
\mid\left(\lambda_{3}+\right. & \left.r-\frac{2 r x_{2}}{x_{M}}+\beta_{1} v_{2}+\beta_{2} y_{2}\right)\left(\lambda_{3}+a\right)\left[\lambda_{3}^{2}+\left(u+p z_{2}\right) \lambda_{3}+p z_{2} c v_{2}\right]\left(\beta_{1} v_{2}+\beta_{2} y_{2}\right) \mid \\
& >\left|\left(\lambda_{3}+r-\frac{2 r x_{2}}{x_{M}}\right) a e^{-\lambda_{3} \tau_{1}}\left[\beta_{2} y_{2}\left(\lambda_{3}^{2}+\left(u+p z_{2}\right) \lambda_{3}+p z_{2} c v_{2}\right)+k \beta_{1} y_{2} \lambda_{3}\right]\right| .
\end{aligned}
$$

Which contradicts to (3.1). Therefore, all roots of equation (3.1) have negative real parts, and $E_{2}$ is locally asymptotically stable.

Theorem 3.2. For any $\tau_{1}, \tau_{2}=0$, the immunity-activated equilibrium $E_{2}$ is globally asymptotically stable if $\Re_{1}>1$.

Proof. Define a Lyapunov functional

$$
\begin{aligned}
V_{3}(t)= & x(t)-x_{2}-x_{2} \ln \frac{x(t)}{x_{2}}+p_{1}\left(y(t)-y_{2}-y_{2} \ln \frac{y(t)}{y_{2}}\right)+p_{2}\left(v(t)-v_{2}-v_{2} \ln \frac{v(t)}{v_{2}}\right) \\
& +p_{3}\left(z(t)-z_{2}-z_{2} \ln \frac{z(t)}{z_{2}}\right)+\beta_{1} x_{2} v_{2} \int_{t-\tau_{1}}^{t}\left[\frac{x(s) v(s)}{x_{2} v_{2}}-1-\ln \frac{x(s) v(s)}{x_{2} v_{2}}\right] \mathrm{d} s \\
& +\beta_{2} x_{2} y_{2} \int_{t-\tau_{1}}^{t}\left[\frac{x(s) y(s)}{x_{2} y_{2}}-1-\ln \frac{x(s) y(s)}{x_{2} y_{2}}\right] \mathrm{d} s
\end{aligned}
$$

here $p_{1}=\frac{1}{\alpha}, \quad p_{2}=\frac{\beta_{1} x_{2}}{u+p z_{2}}, \quad p_{3}=\frac{\beta_{1} p x_{2}}{c u+c p z_{2}}$.

Calculating the derivative of $V(t)$ along the solution of (1.2), we obtain that

$$
\begin{aligned}
\frac{\mathrm{d} V_{3}(t)}{\mathrm{d} t}= & -r \frac{\left(x(t)-x_{2}\right)^{2}}{x_{M}}-\beta_{1} x_{2} v_{2}\left(\frac{x_{2}}{x(t)}-1-\ln \frac{x_{2}}{x(t)}\right) \\
& -\beta_{1} x_{2} v_{2}\left(\frac{y(t) v_{2}}{v(t) y_{2}}-1-\ln \frac{y(t) v_{2}}{v(t) y_{2}}\right)-\beta_{2} x_{2} y_{2}\left(\frac{x_{2}}{x(t)}-1-\ln \frac{x_{2}}{x(t)}\right) \\
& -\beta_{1} x_{2} v_{2}\left(\frac{x\left(t-\tau_{1}\right) v\left(t-\tau_{1}\right) y_{2}}{x_{2} v_{2} y(t)}-1-\ln \frac{x\left(t-\tau_{1}\right) v\left(t-\tau_{1}\right) y_{2}}{x_{2} v_{2} y(t)}\right) \\
& -\beta_{2} x_{2} y_{2}\left(\frac{x\left(t-\tau_{1}\right) y\left(t-\tau_{1}\right)}{x_{2} y(t)}-1-\ln \frac{x\left(t-\tau_{1}\right) y\left(t-\tau_{1}\right)}{x_{2} y(t)}\right) .
\end{aligned}
$$

Since function $g(x)=x-1-\ln x$ is always positive except for $x=1$ where $g(x)=0$. It follows from (3.2) that $\frac{\mathrm{d} V_{3}(t)}{\mathrm{d} t} \leq 0$ with equality holding if and only if $x=x_{2}, y=y_{2}, v=v_{2}, z=z_{2}$. It can be certified that $M_{2}=\left\{E_{2}\right\} \subset \Omega$ is the largest invariant subset of $\left\{(x(t), y(t), v(t), z(t)): \frac{\mathrm{d} V_{2}}{\mathrm{~d} t}=0\right\}$. According to Theorem $3.1, E_{1}$ is locally asymptotically stable. From LaSalles invariance principle, we obtain that $E_{2}$ is globally asymptotically stable. 
In the following, we suppose $\tau_{2}>0$, and devote to investigating the existence of the Hopf bifurcation. The characteristic equation of system (1.2) at the immunity-activated equilibrium $E_{2}$ is

$$
\begin{aligned}
& \lambda^{4}+A_{1} \lambda^{3}+A_{2} \lambda^{2}+A_{3} \lambda+A_{4}+\left(B_{1} \lambda^{3}+B_{2} \lambda^{2}+B_{3} \lambda+B_{4}\right) e^{-\lambda \tau_{2}} \\
& +\left(C_{1} \lambda^{3}+C_{2} \lambda^{2}+C_{3} \lambda+C_{4}\right) e^{-\lambda \tau_{1}}+\left(D_{1} \lambda^{2}+D_{2} \lambda+D_{3}\right) e^{-\lambda \tau_{2}-\lambda \tau_{1}}=0
\end{aligned}
$$

where

$$
\begin{aligned}
& A_{1}=-\left(a_{22}+a_{33}+a_{11}\right) \\
& A_{3}=-a_{11} a_{22} a_{33} \\
& B_{1}=-b_{22} \\
& A_{2}=a_{22} a_{33}+a_{11} a_{33}+a_{11} a_{22} \\
& B_{3}=a_{11} a_{33} b_{23}-a_{11} a_{33} b_{22}-a_{32} a_{13} b_{21} \\
& A_{4}=-a_{11}+a_{22} a_{44} c_{22} \\
& B_{2}=a_{33} b_{22}-a_{32} b_{33}+a_{11} b_{22}-b_{21} a_{12} \\
& B_{4}=-\left(a_{11} a_{33} a_{22} c_{44}\right) \\
& C_{1}=-a_{34} \quad C_{2}=a_{33} a_{34}-a_{34} c_{43}+a_{22} a_{34}+a_{11} a_{34} \\
& C_{3}=a_{22} a_{34} c_{43}-a_{22} a_{34} a_{33}-a_{11} a_{33} a_{34}+a_{11} a_{34} c_{43}-a_{11} a_{22} a_{34} \\
& C_{4}=a_{11} a_{22} a_{34} a_{33}-a_{11} a_{22} a_{34} c_{43} \\
& D_{1}=a_{34} b_{22} \quad D_{2}=a_{34} a_{43} b_{22}+a_{32} a_{34} b_{23}-a_{33} a_{34} b_{22}-a_{11} a_{34} b_{22} \\
& D_{3}=a_{11} a_{33} a_{34} b_{22}-a_{11} a_{34} c_{43} b_{22}-a_{11} a_{32} a_{34} b_{23}+a_{12} a_{34} b_{21}-a_{12} a_{33} a_{34} b_{21} \\
& +a_{12} a_{34} b_{21} c_{43}+a_{32} a_{13} a_{34} b_{21} \text {. }
\end{aligned}
$$

and

$$
\begin{array}{llll}
a_{11}=r-\frac{2 r x_{2}}{x_{M}}-\beta_{1} v_{2}-\beta_{2} y_{2} & a_{12}=-\beta_{2} x_{2} & a_{13}=-\beta_{1} x_{2} & a_{22}=a \\
a_{32}=k & a_{33}=-a-p z_{2} & a_{34}=-p v_{2} & a_{44}=-b \\
b_{21}=\alpha \beta_{1} v_{2}+\alpha \beta_{2} y_{2} & b_{22}=\alpha \beta_{2} x_{2} & b_{23}=\alpha \beta_{1} x_{2} & c_{43}=c z_{2} \\
c_{44}=c v_{2} & & &
\end{array}
$$

We consider (3.3) with $\tau_{1}$ in its stable interval and $\tau_{2}$ is considered as a parameter. For simplification, we consider (3.3) with $\tau_{1}=0$ and $\tau_{2}>0$. Then equation (3.3) is reduced to

$$
\lambda^{4}+E_{1} \lambda^{3}+E_{2} \lambda^{2}+E_{3} \lambda+E_{4}+\left(F_{1} \lambda^{3}+F_{2} \lambda^{2}+F_{3} \lambda+F_{4}\right) e^{-\lambda \tau_{2}}=0,
$$

where

$$
\begin{array}{llll}
E_{1}=A_{1}+C_{1}, & E_{2}=A_{2}+C_{2}, & E_{3}=A_{3}+C_{3}, & E_{4}=A_{4}+C_{4}, \\
F_{1}=B_{1}, & F_{2}=B_{2}+D_{1}, & F_{3}=B_{3}+D_{2}, & F_{4}=B_{4}+D_{3} .
\end{array}
$$

We consider

$$
P(\lambda)+Q(\lambda) e^{-\lambda \tau_{2}}=0
$$

where

$$
\begin{aligned}
& P(\lambda)=\lambda^{4}+E_{1} \lambda^{3}+E_{2} \lambda^{2}+E_{3} \lambda+E_{4} \\
& Q(\lambda)=F_{1} \lambda^{3}+F_{2} \lambda^{2}+F_{3} \lambda+F_{4} .
\end{aligned}
$$

In the following, we investigate the existence of purely imaginary roots $\lambda=i \omega(\omega>0)$ to equation (3.5). Beretta and Kuang [2] established a geometrical criterion which gives the existence of purely imaginary root of a characteristic equation with delay dependent coefficients. 
In order to apply the criterion due to Beretta and Kuang [2], we need to verify the following properties for all $\tau_{2} \in\left[0, \tau_{2 \max }\right)$, where $\tau_{2 \max }$ is the maximum value which $E_{2}$ exists.

(a) $P(0)+Q(0) \neq 0$.

(b) $P(i \omega)+Q(i \omega) \neq 0$.

(c) $\lim \sup \left\{\left|\frac{Q(\lambda)}{P(\lambda)}\right|:|\lambda| \rightarrow \infty \cdot \operatorname{Re} \lambda \geq 0\right\}<1$.

(d) $F(\omega)=|P(i \omega)|^{2}-|Q(i \omega)|^{2}$ has a finite number of zeros.

(e) Each positive root $\omega$ of $F(\omega)=0$ is continuous and differentiable in $\tau_{2}$ whenever it exists.

Here, $P(\lambda)$ and $Q(\lambda)$ are defined as in (3.6).

Let's $\tau \in\left[0, \tau_{2 \max }\right)$. It is easy to see that $P(0)+Q(0)=E_{4}+F_{4} \neq 0$. This implies that (a) is satisfied. And (b) is obviously true because

$$
P(i \omega)+Q(i \omega)=\left[\omega^{4}-\left(E_{2}+F_{2}\right) \omega^{2}+E_{4}+E_{4}\right]+i \omega\left[-\left(E_{1}+F_{3}\right) \omega^{2}+E_{3}-F_{3}\right] \neq 0 .
$$

From (3.6) we know that

$$
\begin{aligned}
\lim _{|\lambda| \rightarrow \infty}\left|\frac{Q(\lambda)}{P(\lambda)}\right| & =\lim _{|\lambda| \rightarrow \infty}\left|\frac{F_{1} \lambda^{3}+F_{2} \lambda^{2}+F_{3} \lambda+F_{4}}{\lambda^{4}+E_{1} \lambda^{3}+E_{2} \lambda^{2}+E_{3} \lambda+E_{4}}\right| \\
& =\lim _{|\lambda| \rightarrow \infty}\left|\frac{3 F_{1} \lambda^{2}+2 F_{2} \lambda+F_{3}}{4 \lambda^{3}+3 E_{1} \lambda^{2}+2 E_{2} \lambda+E_{3}}\right| \\
& =\lim _{|\lambda| \rightarrow \infty}\left|\frac{6 F_{1} \lambda+2 F_{2}}{12 \lambda^{2}+6 E_{1} \lambda+2 E_{2}}\right|=0 .
\end{aligned}
$$

Therefore (c) follows.

Let $G$ be defined as in (d). From

$$
\begin{aligned}
|P(i \omega)|^{2}= & \omega^{8}+\left(E_{1}-2 E_{2}\right) \omega^{6}+\left(E_{2}^{2}+2 E_{4}-2 E_{3} E_{1}\right) \omega^{4} \\
& +\left(E_{3}^{2}-2 E_{4} E_{2}\right) \omega^{2}+E_{4}^{2}, \\
|Q(i \omega)|^{2}= & F_{1}^{2} \omega^{6}+\left(F_{2}^{2}-2 F_{3} F_{1}\right) \omega^{4}+\left(F_{3}^{2}-2 F_{4} F_{2}\right) \omega^{2}+F_{4}^{2} .
\end{aligned}
$$

we have

$$
F(\omega)=\omega^{8}+P_{1} \omega^{6}+P_{2} \omega^{4}+P_{3} \omega^{2}+P_{4}
$$

where $P_{1}=E_{1}-2 E_{2}-F_{1}^{2}, \quad P_{2}=E_{2}^{2}+2 E_{4}-2 E_{3} E_{1}-F_{2}^{2}+2 F_{3} F_{1}$, $P_{3}=E_{3}^{2}-2 E_{4} E_{2}-F_{3}^{2}+2 F_{4} F_{2}, \quad P_{4}=E_{4}^{2}-F_{4}^{2}$.

Let $\omega^{2}=h$, then we have

$$
G(h)=h^{4}+P_{1} h^{3}+P_{2} h^{2}+P_{3} h+P_{4}=0 .
$$

Theorem 3.3. Suppose that (3.7) has no positive roots, then when $\Re_{1}>1, E_{2}$ is locally asymptotically stable for all $\tau_{2} \geq 0$.

Suppose that (3.7) has positive roots, then (d) is satisfied. Let $\omega_{0}$ be a point of its domain of definition such that $F\left(\omega_{0}\right)=0$. We know the partial derivatives $F_{\omega}$ and $F_{\tau 2}$ exist and are continuous in a certain neighborhood of $\omega_{0}$, and $F\left(\omega_{0}\right) \neq 0$. By Implicit Function Theorem, (e) is also satisfied. 
Now let $\lambda=i \omega(\omega>0)$ be a root of equation (3.11), and from which we have that

$$
\omega^{4}-i E_{1} \omega^{3}-E_{2} \omega^{2}+i E_{3} \omega+E_{4}+\left[-i F_{1} \omega^{3}-F_{2} \omega^{2}+i F_{3} \omega+F_{4}\right] e^{-i \omega \tau_{2}}=0 .
$$

Hence, we have

$$
\begin{aligned}
& F_{1} \omega^{3}-F_{3}(\omega) \sin \omega \tau_{2}+\left(F_{2} \omega^{2}-F_{4}\right) \cos \omega \tau_{2}=\omega^{4}-E_{2} \omega^{2}+E_{4} \\
& F_{1} \omega^{3}-F_{3}(\omega) \cos \omega \tau_{2}-\left(F_{2} \omega^{2}-F_{4}\right) \sin \omega \tau_{2}=-E_{1} \omega^{3}+E_{3} \omega
\end{aligned}
$$

From (3.8) it follows that

$$
\begin{aligned}
\sin \omega \tau_{2} & =\frac{\left(\omega^{4}-E_{2} \omega^{2}+E_{4}\right)\left(F_{1} \omega^{3}-F_{3}-\left(-E_{1} \omega^{3}+E_{3} \omega\right)\left(F_{2} \omega^{2}-F_{4}\right)\right.}{\left(F_{1} \omega^{3}-F_{3} \omega\right)^{2}+\left(F_{2} \omega^{2}-F_{4}\right)^{2}}, \\
\cos \omega \tau_{2} & =\frac{\left(\omega^{4}-E_{2} \omega^{2}+E_{4}\right)\left(F_{2} \omega^{2}-F_{4}\right)+\left(-E_{1} \omega^{3}+E_{3} \omega\right)\left(F_{1} \omega^{3}-F_{3} \omega\right)}{\left(F_{1} \omega^{3}-F_{3} \omega\right)^{2}+\left(F_{2} \omega^{2}-F_{4}\right)^{2}} .
\end{aligned}
$$

By the definitions of $P(\lambda), Q(\lambda)$ as in (3.6), and applying the property (a), (3.9) can be written as

$$
\sin \omega \tau_{2}=\operatorname{Im} \frac{P(i \omega)}{Q(i \omega)}, \quad \cos \omega \tau_{2}=-\operatorname{Re} \frac{P(i \omega)}{Q(i \omega)},
$$

which yields

$$
|P(i \omega)|^{2}=|Q(i \omega)|^{2}
$$

Assume that $I \in \mathbb{R}_{+0}$ is the set where $\omega$ is a positive root of

$$
F(\omega)=|P(i \omega)|^{2}-|Q(i \omega)|^{2}=0,
$$

and for $\tau_{2} \neg \in \mathrm{I}, \omega$ is not defined. Then for all $\tau_{2}$ in I, $\omega$ satisfied

$$
F(\omega)=0 .
$$

Assume that equation (3.7) has only one positive real root, we denote by $h_{+}$this positive real root. Thus, equation (3.11) has only one positive real root $\omega=\sqrt{h_{+}}$. And the critical values of $\tau_{2}$ and $\omega$ are impossible to solve explicitly, so we shall use the procedure described in Beretta and Kuang [2]. According to this procedure, we define such that sin and cos are given by (3.10).

And the relation between the argument $\theta$ and $\omega \tau_{2}$ in (3.9) for $\tau_{2}>0$ must be

$$
\omega \tau_{2}=\theta+2 n \pi, \quad n=0,1,2, \ldots
$$

Hence we can define the maps given by

$$
\tau_{n}\left(\tau_{2}\right)=\frac{\theta\left(\tau_{2}\right)+2 n \pi}{\omega\left(\tau_{2}\right)}, \quad \tau_{n}>0, n=0,1,2, \ldots
$$

where a positive root $\theta\left(\tau_{2}\right)$ of (3.7) exists in $I$.

Let us introduce the functions $S_{n}\left(\tau_{2}\right): I \rightarrow \mathbb{R}$,

$$
S_{n}\left(\tau_{2}\right)=\tau_{2}-\frac{\theta\left(\tau_{2}\right)+2 n \pi}{\omega\left(\tau_{2}\right)}, \quad \tau_{n}>0, n=0,1,2, \ldots
$$


that are continuous and differentiable in $\tau_{2}$. Thus, we give the following theorem which is due to Beretta and Kuang [2].

Theorem 3.4. Assume that $\omega\left(\tau_{2}\right)$ is a positive root of (3.11) defined for $\tau_{2}^{*} \in I, I \subseteq \mathbb{R}_{+0}$, and at some $\tau_{2}^{*} \in$ $I, S_{n}\left(\tau_{2}^{*}\right)$ for some $n \in N_{0}$. Then a pair of simple conjugate pure imaginary roots $\lambda=i \omega$ exists at $\tau_{2}=\tau_{2}^{*}$ which crosses the imaginary axis from left to right if $\delta\left(\tau_{0}\right)>0$ and crosses the imaginary axis from right to left if $\delta\left(\tau_{0}\right)<0$, where

$$
\delta\left(\tau_{2}^{*}\right)=\operatorname{sign}\left\{f_{\omega}^{\prime}\left(\omega \tau_{2}^{*}, \tau_{2}^{*}\right)\right\} \operatorname{sign}\left\{\left.\frac{\mathrm{d} S_{n}\left(\tau_{2}\right)}{\mathrm{d} \tau_{2}}\right|_{\tau_{2}=\tau_{2}^{*}}\right\} .
$$

Applying Theorems 3.3, Theorems 3.4 and the Hopf bifurcation theorem for functional differential equation [9], we can obtain the existence of a Hopf bifurcation as stated in Theorem 3.5.

Theorem 3.5. For system (1.2), then there exists $\tau_{2}$ in $I$, such that the equilibrium $E_{2}$ is asymptotically stable for $0 \leq \tau_{2}<\tau_{2}^{*}$, and becomes unstable for $\tau_{2}$ staying in some right neighborhood of $\tau_{2}^{*}$, with a Hopf bifurcation occurring when $\tau_{2}=\tau_{2}^{*}$.

\section{Direction AND Stability of Hopf Bifurcation}

In this section, we discuss that the direction of the Hopf bifurcation and stability of bifurcating periodic solution at the positive equilibrium $E_{2}=\left(x_{2}, y_{2}, v_{2}, z_{2}\right)$ for $\tau_{2}=\tau_{2}^{*}$ by applying the normal form and the center manifold theorem introduced by $[9,10]$. We assume that $\tau_{1}^{*} \geq 0$.

Let $\tau_{2}=\tau_{2}^{*}+\mu, t=s \tau_{2}, u_{1}=x-x_{2}, u_{2}=y-y_{2}, u_{3}=v-v_{2}, u_{4}=z-z_{2}$. Still denoting $s=t$, then system (1.2) can be transformed into the following form

$$
\frac{\mathrm{d} u(t)}{\mathrm{d} t}=L_{\mu}\left(u_{t}\right)+f\left(\mu, u_{t}\right)
$$

where $u(t)=\left(u_{1}, u_{2}, u_{3}, u_{4}\right)^{T} \in \mathbb{C}=\mathbb{C}\left([-1,0], \mathbb{R}^{4}\right)$, and $L_{\mu}: \mathbb{C} \rightarrow \mathbb{R}^{4}, \quad f: \mathbb{R} \times \mathbb{C} \rightarrow \mathbb{R}^{4}$, are given, respectively, by

$$
L_{\mu}(\phi)=\left(\tau_{2}^{*}+\mu\right)\left[M_{1} \phi(0)+M_{2} \phi\left(\frac{-\tau_{1}^{*}}{\tau_{2}^{*}}\right)+M_{3} \phi(-1)\right]
$$

and

$$
f(\mu, \phi)=\left(\tau_{2}^{*}+\mu\right)\left(f_{1}, f_{2}, f_{3}, f_{4}\right)^{T}=\left(\tau_{2}^{*}+\mu\right)\left(\begin{array}{c}
-\frac{r}{x_{M}} \phi_{1}^{2}(0)-\beta_{1} \phi_{1}(0) \phi_{3}(0)-\beta_{2} \phi_{1}(0) \phi_{2}(0) \\
\beta_{1} \alpha \phi_{1}(0) \phi_{3}(0)+\beta_{2} \alpha \phi_{1}(0) \phi_{3}(0) \\
-p \phi_{3}(0) \phi_{4}(0) \\
c \phi_{3}(-1) \phi_{4}(-1)
\end{array}\right)
$$

with

$$
\begin{aligned}
& M_{1}=\left(\begin{array}{cccc}
r-\frac{2 r x_{2}}{x_{M}}-\beta_{1} v_{2}-\beta_{2} y_{2} & -\beta_{2} x_{2} & -\beta_{1} x_{2} & 0 \\
0 & -a & 0 & 0 \\
0 & k & -u-p x_{2} & -p v_{2} \\
0 & 0 & 0 & -b
\end{array}\right) \text {, } \\
& M_{2}=\left(\begin{array}{cccc}
0 & 0 & 0 & 0 \\
\beta_{1} \alpha v_{2}+\beta_{2} \alpha y_{2} & \beta_{2} \alpha x_{2} & \beta_{1} \alpha x_{2} & 0 \\
0 & 0 & 0 & 0 \\
0 & 0 & 0 & 0
\end{array}\right), \quad M_{3}=\left(\begin{array}{cccc}
0 & 0 & 0 & 0 \\
0 & 0 & 0 & 0 \\
0 & 0 & 0 & 0 \\
0 & 0 & c z_{2} & c v_{2}
\end{array}\right) \text {. }
\end{aligned}
$$


Therefore, according to the Riesz representation theorem, there exists a $4 \times 4$ matrix function $\eta(\theta, \mu)$ : $[-1,0] \rightarrow \mathbb{R}^{4}$ whose elements are of bounded variations such that

$$
L_{\mu}(\phi)=\int_{-1}^{0} \mathrm{~d} \eta(\theta, \mu) \phi(\theta), \quad \text { for } \phi \in \mathbb{C} .
$$

Here, we can choose

$$
\eta(\theta, \mu)= \begin{cases}\left(\tau_{2}^{*}+\mu\right)\left(M_{1}+M_{2}+M_{3}\right), & \theta=0, \\ \left(\tau_{2}^{*}+\mu\right)\left(M_{2}+M_{3}\right), & \theta \in\left[\frac{-\tau_{*}^{*}}{\tau_{2}^{*}}, 0\right), \\ \left(\tau_{2}^{*}+\mu\right) M_{3}, & \theta \in\left(-1, \frac{-\tau_{1}^{*}}{\tau_{2}^{*}}\right), \\ 0 & \theta=-1 .\end{cases}
$$

For any $\phi \in \mathbb{C}$, we define that

$$
\left(A_{\mu} \phi\right)(\theta)= \begin{cases}\frac{\mathrm{d} \phi(\theta)}{\mathrm{d} \theta}, & \theta \in[-1,0), \\ \int_{-1}^{0} \mathrm{~d} \eta(\theta, \mu) \phi(\theta), & \theta=0 .\end{cases}
$$

and

$$
\left(R_{\mu} \phi\right)(\theta)= \begin{cases}0, & \theta \in[-1,0) \\ F(\phi, \theta) . & \theta=0\end{cases}
$$

Next, system (4.1) is equivalent to the following operator equation

$$
\frac{\mathrm{d} u_{t} \theta}{\mathrm{d} t}=A_{u} u_{t}+R(\mu) u_{t}
$$

with $u_{t}(\theta)=u(t+\theta), \quad \theta \in[-1,0]$. The adjoint operator $A^{*}$ of $A(0)$ is defined by for $\varphi \in \mathbb{C}\left([0,1],\left(\mathbb{R}^{4}\right)^{*}\right)$, define

$$
\left(A^{*} \varphi\right)(s)= \begin{cases}-\frac{\mathrm{d} \varphi(s)}{\mathrm{d} s}, & s \in(0,1], \\ \int_{-1}^{0} \mathrm{~d} \eta^{T}(s, 0) \varphi(-s), & s=0,\end{cases}
$$

and a bilinear inner product

$$
<\varphi(s), \phi(\theta)>=\bar{\varphi}(0) \phi(0)-\int_{\theta=-1}^{0} \int_{\xi=0}^{\theta} \bar{\varphi}(\xi-\theta) \mathrm{d} \eta(\theta) \phi(\xi) \mathrm{d} \xi
$$

where $\eta(\theta)=\eta(\theta, 0), A_{0}$ and $A^{*}$ are adjoint operators. We know that $\pm i \omega_{0} \tau_{0}$ are the eigenvalue of $A_{0}$. Then are also the eigenvalue of $A^{*}$.

Let $q(\theta)=\left(1, q_{2}, q_{3}, q_{4}\right)^{T} e^{\omega_{2}^{*} \tau_{2}^{*} \theta}$ be the eigenvector of $A_{0}$ corresponding to $i \omega_{2}^{*} \tau_{2}^{*}$, thus $A_{0} q(\theta)=i \omega_{2}^{*} \tau_{2}^{*} q(\theta)$, then on the basis of the definition of $A_{0}$ and (4.2)-(4.4), when $\theta=0$, we have

$$
\left(\begin{array}{cccc}
d_{11} & -\beta_{2} x_{2} & -\beta_{1} x_{2} & 0 \\
d_{21} & d_{22} & d_{23} & 0 \\
0 & k & d_{33} & -p v_{2} \\
0 & 0 & d_{43} & d_{44}
\end{array}\right)\left(\begin{array}{c}
1 \\
q_{2} \\
q_{3} \\
q_{4}
\end{array}\right)=\left(\begin{array}{l}
0 \\
0 \\
0 \\
0
\end{array}\right)
$$


where

$$
\begin{array}{lll}
d_{11}=r-\frac{2 r x_{2}}{x_{M}}-\beta_{1} v_{2}-\beta_{2} y_{2}-i w_{2}^{*}, & d_{21}=\alpha e^{-i \omega_{2}^{*}}\left(\beta_{1} v_{2}+\beta_{2} y_{2}\right), \\
d_{22}=\beta_{2} \alpha e^{-i \omega_{2}^{*}} x_{2}-a-i w_{2}^{*}, & d_{23}=\beta_{1} \alpha e^{-i \omega_{2}^{*}} x_{2}, \\
d_{33}=-u-p z_{2}-i w_{2} *, & d_{43}=c z_{2} e^{-i w_{2}^{*} \tau_{2}^{*}} \\
d_{44}=c v_{2} e^{-i w_{2}^{*} \tau_{2}^{*}}-b-i w_{2}^{*} . &
\end{array}
$$

Thus, we can obtain from above

$$
q_{2}=\frac{d_{11}-\beta_{1} x_{2} q_{3}(0)}{\beta_{2} x_{2}}, \quad q_{3}=\frac{d_{22} \beta_{2} x_{2}+d_{21} d_{11}}{d_{21} \beta_{1} x_{2}-\beta_{2} x_{2} d_{23}}, \quad q_{4}=\frac{d_{43} d_{22} \beta_{2} x_{2}+d_{43} d_{21} d_{11}}{\beta_{2} x_{2} d_{23} d_{44}-\beta_{1} x_{2} d_{22} d_{44}} .
$$

Similarly, we suppose that $q^{*}(s)=D\left(1, q_{2}^{*}, q_{3}^{*}, q_{4}^{*}\right)^{T} e^{i \omega_{2}^{*} \tau_{2}^{*} s}$ be the eigenvector of $A^{*}$ corresponding to $-i \omega_{2}^{*} \tau_{2}^{*}$, and $A^{*} q^{*}(s)=-i \omega_{2}^{*} \tau_{2}^{*} q^{*}(s)$, we can calculate that

$$
q_{2}^{*}=\frac{d_{21}}{d_{11}}, \quad q_{3}^{*}=\frac{d_{11} \beta_{2} x_{2}-d_{22} d_{21}}{d_{11} k}, \quad q_{4}^{*}=\frac{d_{11} \beta_{2} p v_{2} x_{2}-d_{22} d_{21} p v_{2}}{d_{44} d_{11} k} .
$$

Here $\left\langle q^{*}, q\right\rangle=1$ and $\left\langle q^{*}, \bar{q}\right\rangle=0$. From (4.6), we can choose that

$$
\bar{D}=\frac{1}{1+q_{2} \bar{q}_{2}^{*}+q_{3} \bar{q}_{3}^{*}+q_{4} \bar{q}_{4}^{*}+\alpha e^{-i \omega_{2}^{*}} \bar{q}_{2}^{*}\left(\beta_{1} v_{2}+\beta_{2} y_{2}+\beta_{2} x_{2} q_{2}+\beta_{1} x_{2} q_{3}\right)+e^{-i w_{2}^{*} \tau_{2}^{*}} \bar{q}_{4}^{*}\left(c z_{2} q_{3}+c v_{2} q_{4}\right)} .
$$

Following the algorithms given in Hassard et al. [10] and using similar computation process in [12], we can get the coefficients which can be used to determine the direction of Hopf bifurcation and the stability of bifurcating periodic solutions denoted by

$$
\begin{aligned}
g_{20}= & 2 \tau_{2}^{*} \bar{D}\left[\left(-\beta_{1} q_{3}-\beta_{2} q_{2}\right)+\left(\beta_{1} e^{-m \tau_{1}^{*}} q_{3}+\beta_{2} e^{-m \tau_{1}^{*}} q_{2}\right) \bar{q}_{2}^{*}-p q_{3} q_{4} \bar{q}_{3}^{*}+c q_{3} q_{4} \bar{q}_{4}^{*} e^{-2 i \tau_{2}^{*} \omega_{2}^{*}}\right] \\
g_{11}= & \tau_{2}^{*} \bar{D}\left[-\beta_{1}\left(q_{3}+\bar{q}_{3}\right)-\beta_{2}\left(q_{2}+\bar{q}_{2}\right)+\left(\beta_{1} e^{-m \tau_{1}^{*}}\left(q_{3}+\bar{q}_{3}\right)+\beta_{2} e^{-m \tau_{1}^{*}}\left(q_{2}+\bar{q}_{2}\right)\right) \bar{q}_{2}^{*}\right. \\
& \left.-p\left(q_{3} \bar{q}_{4}+q_{4} \bar{q}_{3}\right) \bar{q}_{3}^{*}+c\left(q_{3} \bar{q}_{4}+q_{4} \bar{q}_{3}\right) \bar{q}_{4}^{*}\right], \\
g_{02}= & 2 \tau_{2}^{*} \bar{D}\left[\left(-\beta_{1} \bar{q}_{3}-\beta_{2} \bar{q}_{2}\right)+\left(\beta_{1} e^{-m \tau_{1}^{*}} \bar{q}_{3}+\beta_{2} e^{-m \tau_{1}^{*}} \bar{q}_{2}\right) \bar{q}_{2}^{*}-p \bar{q}_{3} \bar{q}_{4} \bar{q}_{3}^{*}+c \bar{q}_{3} \bar{q}_{4} \bar{q}_{4}^{*} e^{-2 i \tau_{2}^{*} \omega_{2}^{*}}\right], \\
g_{21}= & 2 \tau_{2}^{*} \bar{D}\left\{\left[-\frac{r}{x_{M}} 2 W_{11}^{1}(0)-\beta_{1} W_{11}^{3}(0)+q_{3} W_{11}^{1}(0)+\frac{1}{2} W_{20}^{3}(0)+\frac{1}{2} \bar{q}_{3} W_{20}^{1}(0)\right.\right. \\
& \left.-\beta_{2} W_{11}^{2}(0)+q_{2} W_{11}^{1}(0)+\frac{1}{2} W_{20}^{2}(0)+\frac{1}{2} \bar{q}_{2} W_{20}^{1}(0)\right] \\
& +\bar{q}_{2}^{*}\left[\beta_{1} e^{-m \tau_{1}^{*}} W_{11}^{3}(0)+q_{3} W_{11}^{1}(0)+\frac{1}{2} W_{20}^{3}(0)+\frac{1}{2} \bar{q}_{3} W_{20}^{1}(0) \beta_{2} e^{-m \tau_{1}^{*}} W_{11}^{2}(0)\right. \\
& \left.+q_{2} W_{11}^{1}(0)+\frac{1}{2} W_{20}^{2}(0)+\frac{1}{2} \bar{q}_{2} W_{20}^{1}(0)\right] \\
& -p \bar{q}_{3}^{*}\left[q_{3} W_{11}^{4}(0)+q_{4} W_{11}^{3}(0)+\frac{1}{2} \bar{q}_{3} W_{20}^{4}(0)+\frac{1}{2} \bar{q}_{4} W_{20}^{3}(0)\right] \\
& \left.-c \bar{q}_{4}^{*}\left[\left(q_{3} W_{11}^{4}(-1)+q_{4} W_{11}^{3}(-1)\right) e^{-i \tau_{2}^{*}}+\left(\bar{q}_{3} W_{20}^{4}(-1)+\bar{q}_{4} W_{20}^{3}(-1)\right) \frac{e^{-i \tau_{2}^{*} \omega_{2}^{*}}}{2}\right]\right\}
\end{aligned}
$$

with

$$
W_{20}(\theta)=\frac{i g_{20}}{\omega_{2}^{*} \tau_{2}^{*}} q(0) e^{i \omega_{2}^{*} \tau_{2}^{*} \theta}+\frac{i \bar{g}_{02}}{3 \omega_{2}^{*} \tau_{2}^{*}} \bar{q}(0) e^{-i \omega_{2}^{*} \tau_{2}^{*} \theta}+G_{1} e^{2 i \omega_{2}^{*} \tau_{2}^{*} \theta},
$$




$$
W_{11}(\theta)=-\frac{i g_{11}}{\omega_{2}^{*} \tau_{2}^{*}} q(0) e^{i \omega_{2}^{*} \tau_{2}^{*} \theta} \frac{i \bar{g}_{11}}{\omega_{2}^{*} \tau_{2}^{*}} \bar{q}^{*}(0) e^{-i \omega_{2}^{*} \tau_{2}^{*} \theta}+G_{2}
$$

where $G_{1}$ and $G_{2}$ can be computed by the following equations, respectively

$$
\left(\begin{array}{cccc}
b_{11}+2 i w_{2}^{*} & \beta_{2} x^{*} & \beta_{1} x^{*} & 0 \\
-a_{21} & b_{22}+2 i w_{2}^{*} & -a_{23} & 0 \\
0 & -k & u+p z_{2}+2 i w_{2} * & p v_{2} \\
0 & 0 & -a_{43} & b_{44}+2 i w_{2}^{*}
\end{array}\right) G_{1}=2\left(\begin{array}{c}
-\beta_{1} q_{3}-\beta_{2} q_{2} \\
\beta_{1} e^{-m \tau_{1}^{*}} q_{3}+\beta_{2} e^{-m \tau_{1}^{*}} q_{2} \\
-p q_{3} q_{4} \\
c q_{3} q_{4} e^{-2 i \tau_{2}^{*} \omega_{2}^{*}}
\end{array}\right)
$$

and

$$
\left(\begin{array}{cccc}
b_{11} & \beta_{2} x^{*} & \beta_{1} x^{*} & 0 \\
-a_{21} & b_{22} & -a_{23} & 0 \\
0 & -k & u+p z_{2} & p v_{2} \\
0 & 0 & -a_{43} & b_{44}
\end{array}\right) G_{2}=2\left(\begin{array}{c}
-\beta_{1}\left(q_{3}+\bar{q}_{3}\right) \\
\beta_{1} e^{-m \tau_{1}^{*}}\left(q_{3}+\bar{q}_{3}\right)+\beta_{2} e^{-m \tau_{1}^{*}}\left(q_{2}+\bar{q}_{2}\right) \\
-p\left(q_{3} \bar{q}_{4}+q_{4} \bar{q}_{3}\right) \\
-p\left(q_{3} \bar{q}_{4}+q_{4} \bar{q}_{3}\right)
\end{array}\right)
$$

where $b_{11}=d+\beta_{1} v_{2}+\beta_{2} y_{2}, b_{22}=-\beta_{2} e^{-\tau_{1}^{*}\left(m+i w_{2}^{*}\right)} x_{2}+a, b_{44}=-c v_{2} e^{-i w_{2}^{*} \tau_{2}^{*}}+b$. Then we can compute the following values:

$$
\begin{array}{ll}
c_{1}(0)=\frac{i}{2 \omega_{2}^{*} \tau_{2}^{*}}\left(g_{20} g_{11}-2\left|g_{11}\right|^{2}-\frac{\left|g_{02}\right|^{2}}{3}\right)+\frac{g_{21}}{2}, & \mu_{2}=-\frac{\operatorname{Re}\left\{c_{1}(0)\right\}}{\operatorname{Re}\left\{\lambda^{\prime}\left(\tau_{2}^{*}\right)\right\}}, \\
\alpha_{2}=2 \operatorname{Re}\left\{c_{1}(0)\right\}, & T_{2}=-\frac{\operatorname{Im}\left\{c_{1}(0)\right\}+\mu_{2} \operatorname{Im}\left\{\lambda^{\prime}\left(\tau_{2}^{*}\right)\right\}}{\omega_{2}^{*} \tau_{2}^{*}} .
\end{array}
$$

Which determine the qualities of bifurcating periodic solutions in the center manifold at the critical value $\tau_{2}^{*}$.

Theorem 4.1. For the delayed model (1.2), when $\tau_{2}=\tau_{2}^{*}$, the direction and the stability of periodic solution of Hopf bifurcation is determined by (4.7). Then,

(i) the sign of $\mu_{2}$ determines the directions of the Hopf bifurcation: if $\mu_{2}>0\left(\mu_{2}<0\right)$, then the Hopf bifurcation is supercritical (subcritical) and the bifurcation periodic solutions exist for $\tau>\tau_{2}^{*}\left(\tau<\tau_{2}^{*}\right)$;

(ii) the sign of $\alpha_{2}$ determines the stability of the bifurcation periodic solutions: the bifurcation periodic solutions are stable(unstable) if $\alpha_{2}<0\left(\alpha_{2}>0\right)$;

(iii) the sign of $T_{2}$ determines the period of the bifurcation periodic solutions: the period increases (decreases) if $T_{2}>0\left(T_{2}<0\right)$.

\section{NumericAl SIMULATIONS}

In this section, we will give some numerical simulations in order to illustrate the theoretical results obtained in this paper.

We chose the parameter values set as $x_{M}=100000 ; \quad \beta_{1}=4.8 \times 10^{-7} \mathrm{ml}^{-1}$ day $^{-1} ; \quad \beta_{2}=4.7 \times$ $10^{-7} \mathrm{ml}^{-1}$ day $^{-1} ; \quad \alpha=0.9$ day $^{-1} ; \quad a=0.9$ day $^{-1} ; \quad p=0.06$ day $^{-1} ; b=1$ day $^{-1} ; u=13$ day $^{-1} ; c=$ 0.00001 day $^{-1}$ (See Tab. 1).

(1) We consider parameter $r=0.01$ day $^{-1} ; k=70$ day $^{-1}$ and the initial value is $(500,600,2000,50)$. By a simple computation, we get $\Re_{0}=0.5498<1, E_{0}=(100000,0,0,0)$. We consider delay $\tau_{1}=0$ day $^{-1}$, $\tau_{2}=1$ day $^{-1}$ (see Fig. 1), which shows that $E_{0}$ is globally asymptotically stable, and this result supports the result of Theorem 2.2.

(2) We consider parameter $r=0.99$ day $^{-1} ; k=138.44$ day $^{-1}$, and the initial value is $(500,600,2000,50)$. By a simple computation, we get $\Re_{0}=1.0047>1>\Re_{1}=0.9537, E_{1}=(98935,837.23,8927.51,0)$. 
TABLE 1. The values and sources of parameters in the model.

\begin{tabular}{lcc}
\hline Parameters & Values & Source \\
\hline$x_{M}$ & 100000 & {$[17]$} \\
$\beta_{1}$ & $4.8 \times 10^{-7} \mathrm{ml}^{-1} \mathrm{day}^{-1}$ & {$[17]$} \\
$\beta_{2}$ & $4.7 \times 10^{-7} \mathrm{ml}^{-1} \mathrm{day}^{-1}$ & {$[17]$} \\
$\alpha$ & 0.9 day $^{-1}$ & Assumed \\
$a$ & 0.9 day $^{-1}$ & Assumed \\
$p$ & 0.06 day $^{-1}$ & {$[17]$} \\
$b$ & 1 day $^{-1}$ & {$[17]$} \\
$u$ & 13 day $^{-1}$ & {$[17]$} \\
$c$ & 0.00001 day $^{-1}$ & {$[17]$} \\
$r$ & 0.1 & Assumed \\
$k$ & 70 & {$[17]$} \\
\hline
\end{tabular}
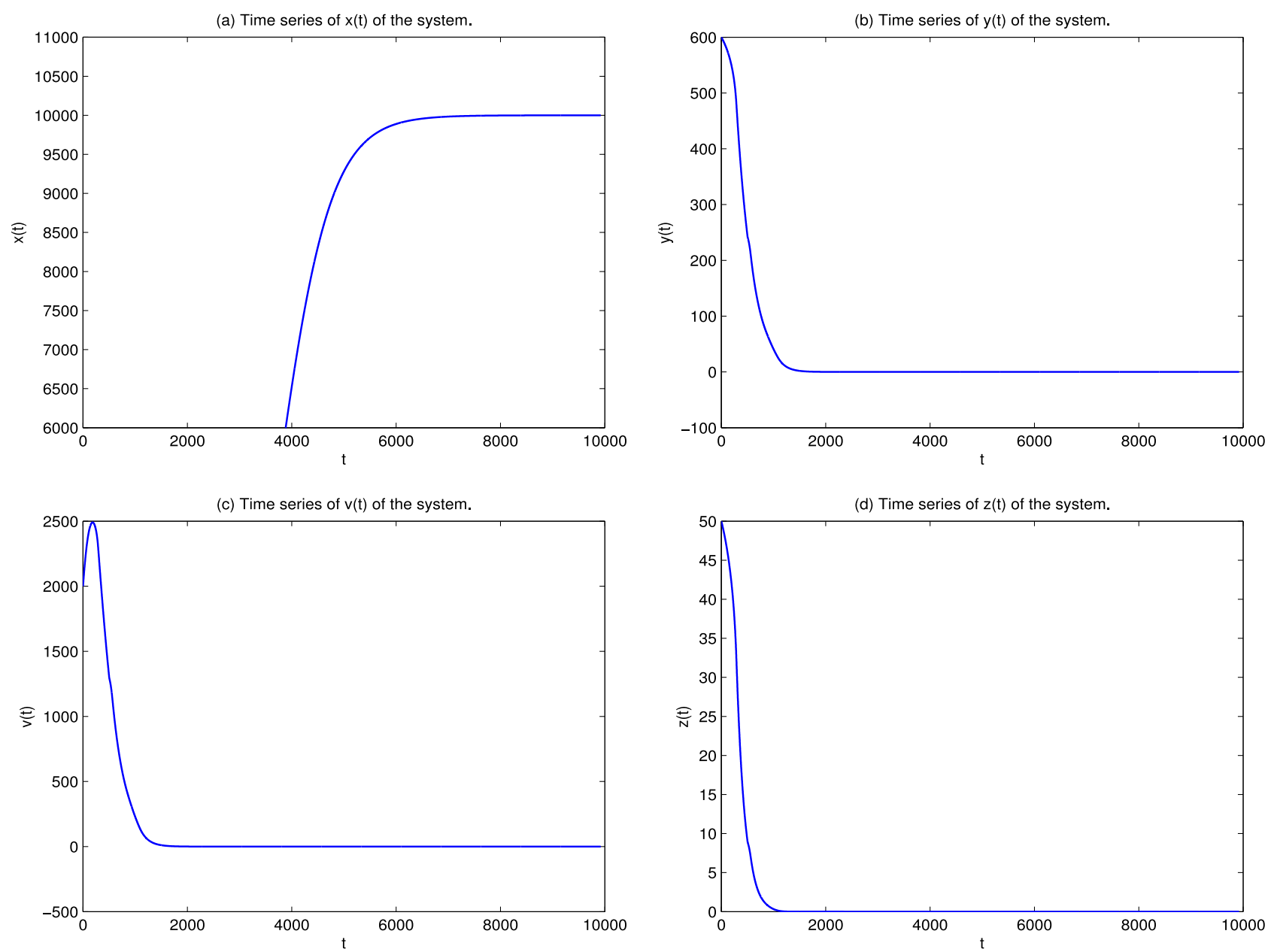

FiguRE 1 . The infection-free equilibrium $E_{0}$ is asymptotically stable for $\tau_{1}=0, \tau_{2}=1$. 

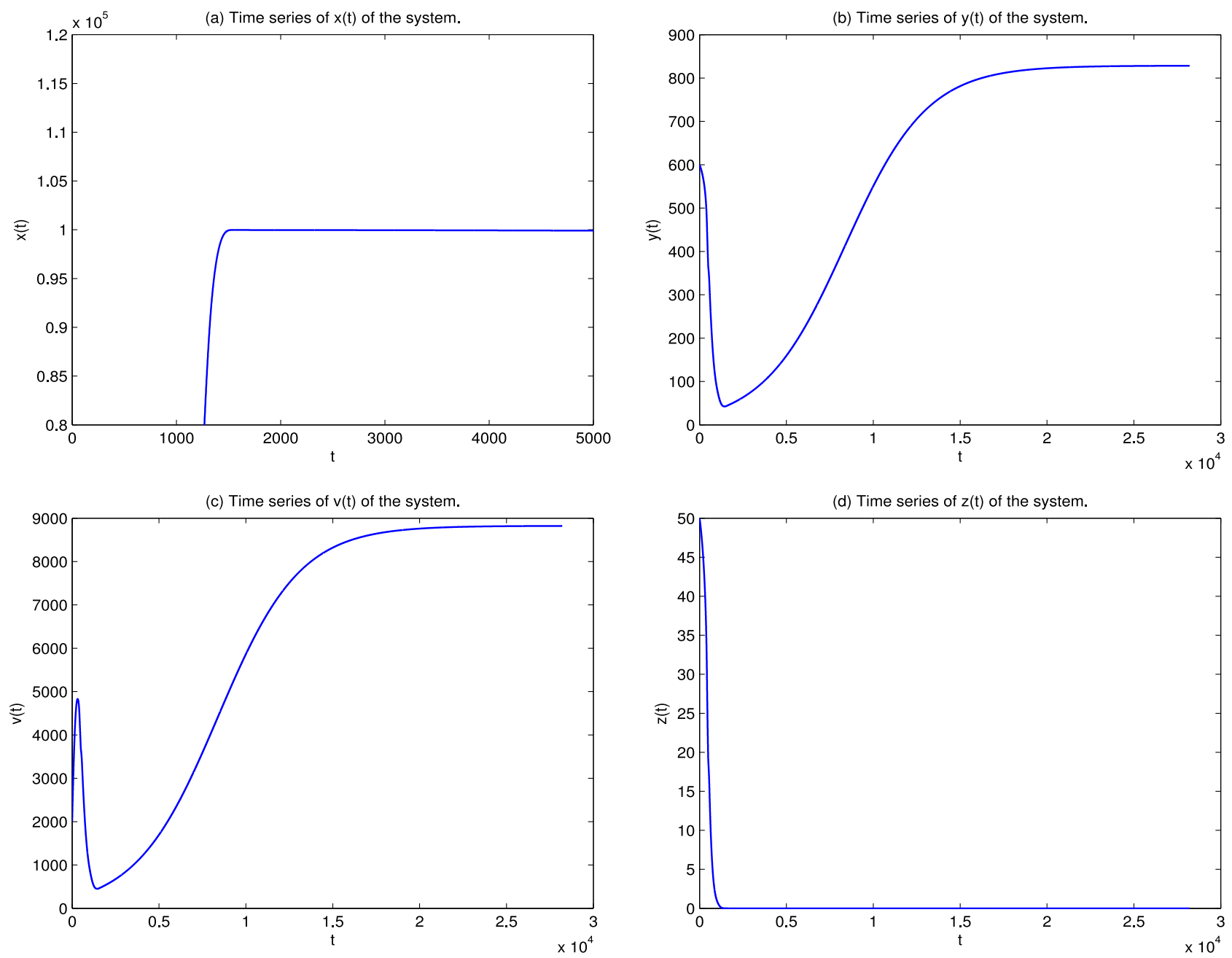

FIGURE 2. The immunity-inactivated equilibrium $E_{1}$ is asymptotically stable for $\tau_{1}=0, \tau_{2}=1$.

We consider delay $\tau_{1}=0$ day $^{-1}, \tau_{2}=1$ day $^{-1}$, from Figure 2 , we can see that the immunity-inactivated equilibrium $E_{1}$ is globally asymptotically stable, which supports the result of Theorem 2.4.

(3) We consider parameter $r=60$ day $^{-1} ; k=170$ day $^{-1}$, and the initial value is $(500,600,2000,50)$. By a simple computation, we get $\Re_{1}=1.2128>1, E_{2}=(98253,10623.71,9852.58,117.53)$, and $\tau_{1}=$ 4.5 day $^{-1}, \tau_{2}=0$ day $^{-1}$. From Figure 3 , we can see that the immunity-activated equilibrium $E_{2}$ is globally asymptotically stable, which supports the result of Theorem 3.2.

(4) We consider parameter $r=60$ day $^{-1} ; k=170$ day $^{-1}$, and the initial value is $(500,600,2000,50)$. By a simple computation, we get $\Re_{1}=1.2128>1, E_{2}=(98253,10623.71,9852.58,117.53)$, and the critical value of delay $\tau_{2}^{*} \approx 3.412$ day $^{-1}$. According to Theorem 3.5, system (1.2) undergoes a Hopf bifurcation at the equilibrium $E_{2}$ when $\tau_{2}=\tau_{2}^{*}$. We chose $\tau_{1}=0$ day $^{-1}, \tau_{2}=3<\tau_{2}^{*}$, then the immune-activated equilibrium $E_{2}$ is asymptotically stable (see Fig. 4 ), we chose $\tau_{1}=0$ day $^{-1}, \tau_{2}=4.5>\tau_{2}^{*}=3.412$ day $^{-1}$, then the equilibrium $E_{2}$ becomes unstable (see Fig. 5). Hence the results obtained in Theorem 3.4 are verified. 

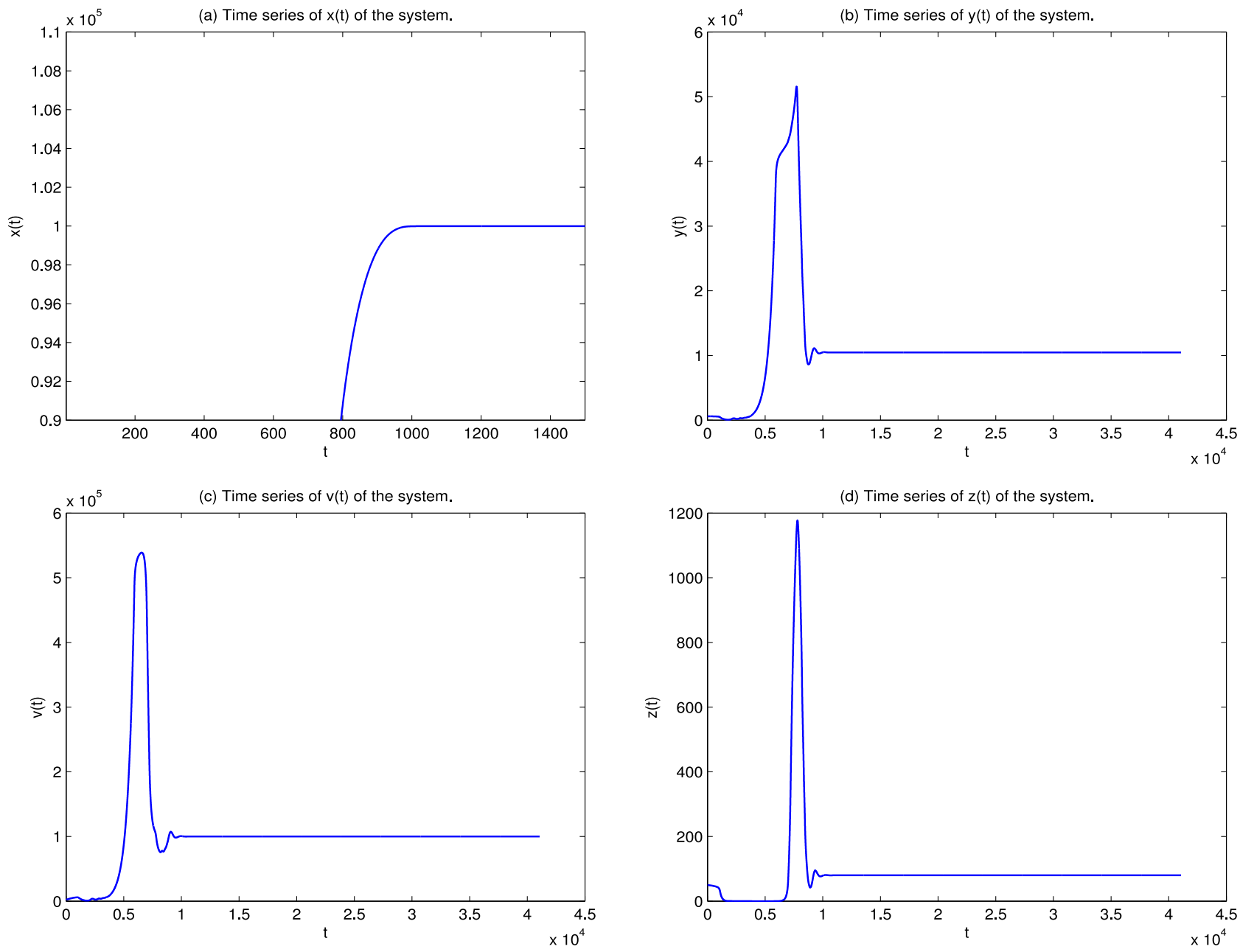

Figure 3 . The immunity-activated equilibrium $E_{2}$ is asymptotically stable for $\tau_{1}=4.5, \tau_{2}=0$.

\section{Conclusions}

In this paper, we have considered two delay HIV-1 virus system to describe both virus-to-cell and cell-to-cell transmissions. Compared with the model mentioned in [17], our model accounts for not only the intracellular delay but also the time delay due to humoral immunity. At the same time, we have considered a HIV-1 infection model with logistic growth for target cells. By a rigorous mathematical analysis,we found that for any $\tau_{1}, \tau_{2}$ the local stability of feasible equilibria is established, it is verified that global stability of the infection-free equilibrium $E_{0}$ and immunity-inactivated equilibrium $E_{1}$. Notice that when $\tau_{2}=0$, the immunity-activated equilibrium $E_{2}$ is global stable which shows that the intracellular delay and logistic growth does not affect the global stability of feasible equilibria. Furthermore, we can see that the humoral immunity delay $\tau_{2}$ plays an important role in the dynamics of model (1.2). The time delay $\tau_{2}$ can change the stability of $E_{2}$ and lead to the existence of Hopf bifurcations when the time delay $\tau_{2}$ exceeds the critical value $\tau_{2}^{*}$. The direction and stability of bifurcating periodic solutions are deduced in explicit formula by using center manifold and normal form method. Clearly, compares to the earlier studies, our analysis combining many aspects is to the actual conditions. This is valuable in the perspectives of biology. Finally, numerical simulations vividly illustrate our main results of model (1.2). Time delays are not the only factors affecting the spread of infectious diseases. 
For example, to optimize the control of diseases by means of vaccine prevention and treatment, and establish models, which will be our future research content.
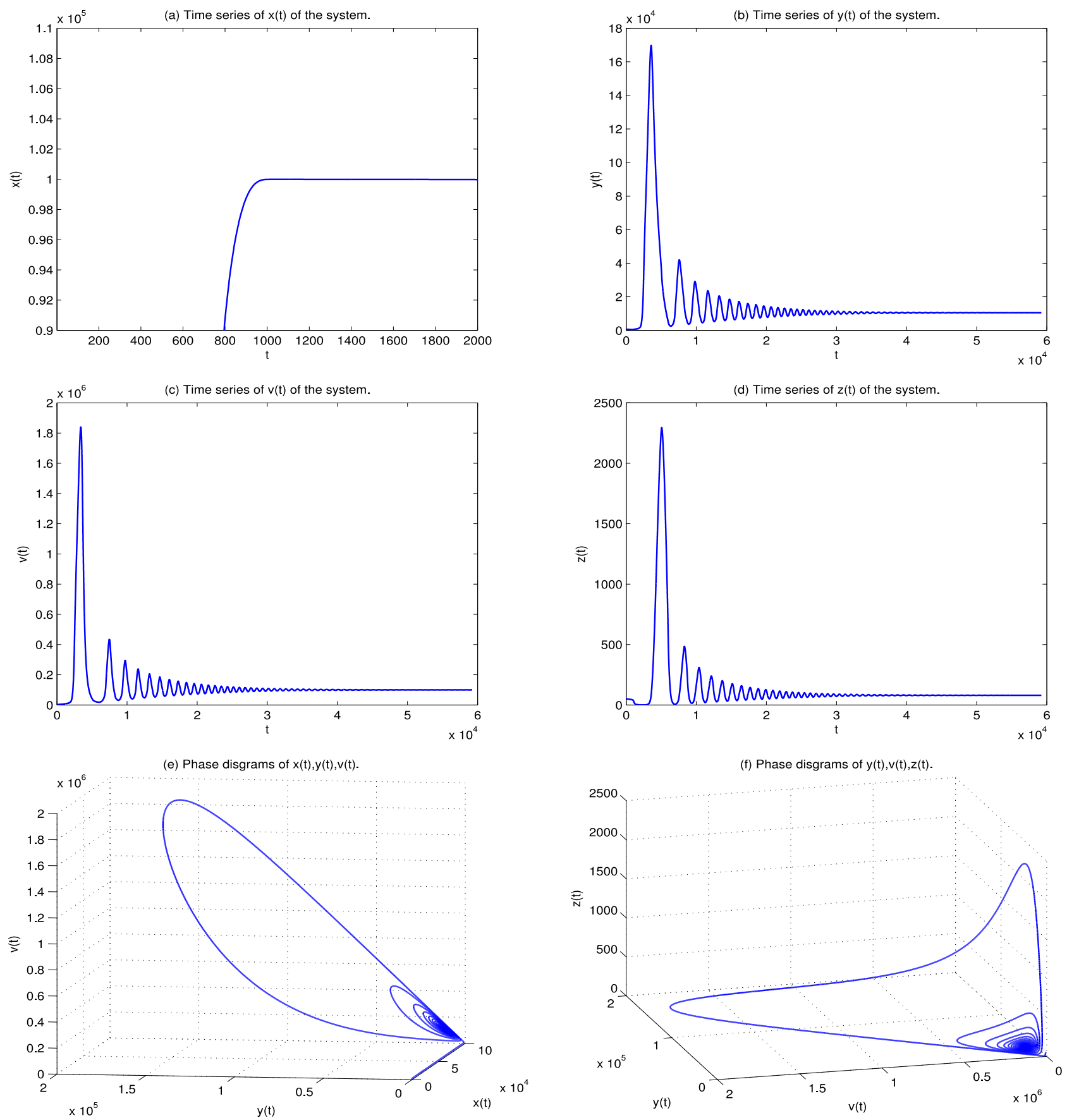

FiguRE 4. The immune-activated equilibrium $E_{2}$ is asymptotically stable when $\tau_{1}=0$, $\tau_{2}=3<\tau_{2}^{*}$. 

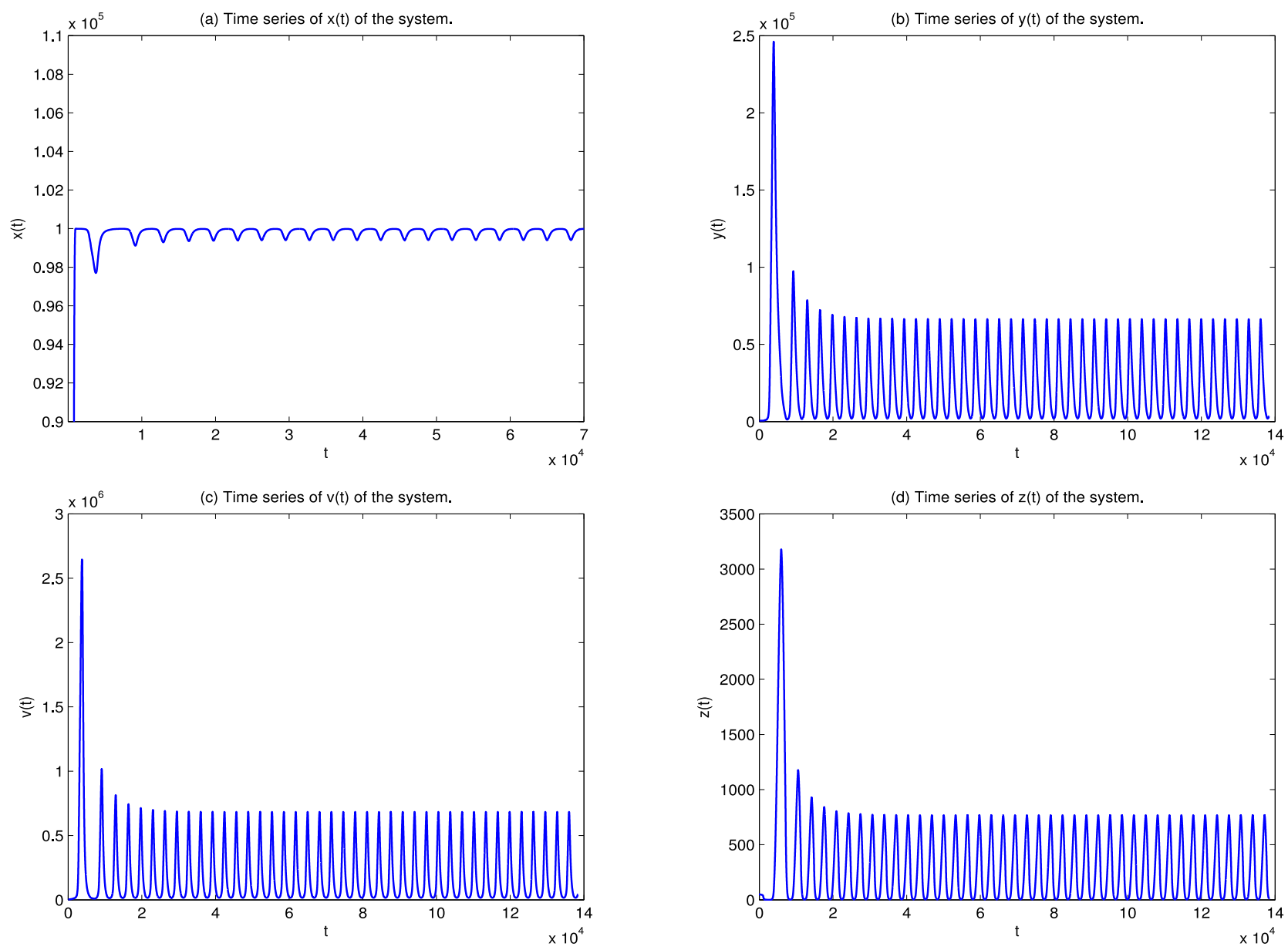

(e) Phase disgrams of $x(t), y(t), v(t)$.

(f) Phase disgrams of $y(t), v(t), z(t)$.
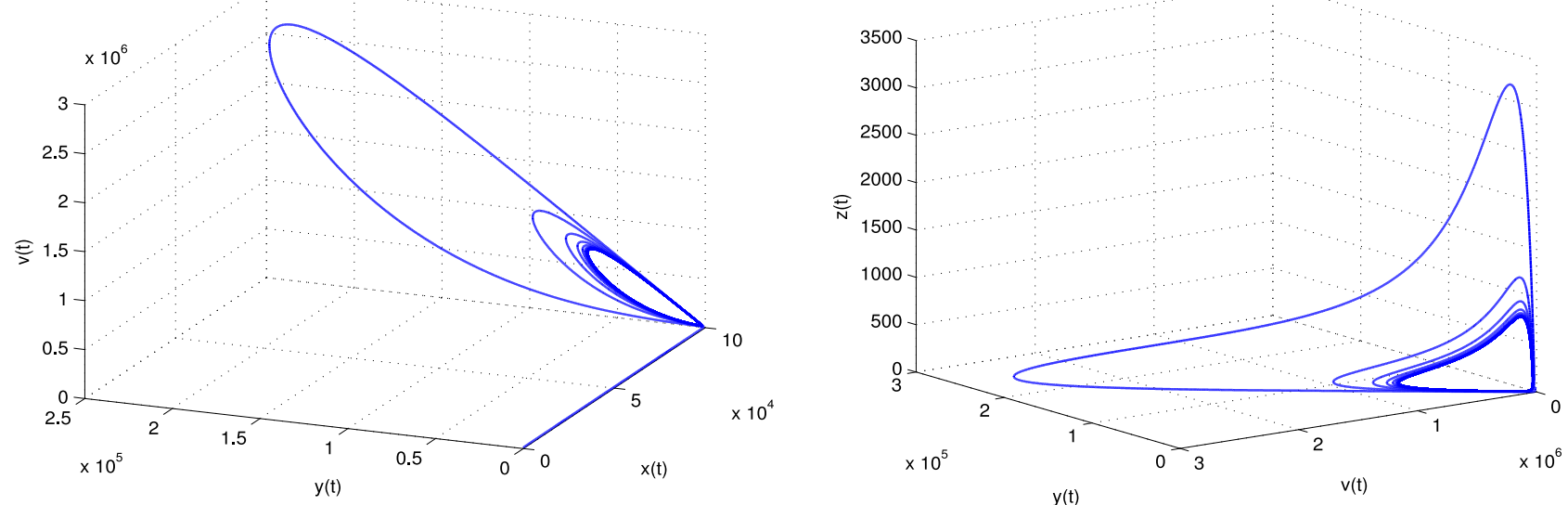

Figure 5. The immune-activated equilibrium $E_{2}$ is unstable when $\tau_{1}=0, \tau_{2}=4.5>\tau_{2}^{*}$. 


\section{REFERENCES}

[1] E. Avila-Vales, N. Chan-Chi, G. E. Garcia-Almeida and C. Vargas-De-Leon, Stability and Hopf bifurcation in a delayed viral infection model with mitosis transmission. Appl. Math. Comput. 259 (2015) 293-312.

[2] E. Beretta and Y. Kuang, Geometric stability switch criteria in delay differential systems with delay dependent parameters. SIAM J. Math. Anal. 33 (2002) 1144-1165.

[3] A.A. Canabarro, I.M. Gl'eria and M.L. Lyra, Periodic solutions and chaos in a non-linear model for the delayed cellular immune response. Physica A 342 (2004) 234-241.

[4] M.S. Ciupe, B.L. Bivort, D.M. Bortz and P.W. Nelson, Estimating kinetic paraneters from HIV primary infection data through the eyes of three different mathematical models. Math. Biosci. 200 (2006) 1-27.

[5] R.V. Culshaw, S. Ruan and G. Webb, A mathematical model of cell-to-cell spread of HIV-1 that includes a time delay. J. Math. Biol. 46 (2003) 425-444.

[6] R.J. De Boer and A.S. Perelson, Target cell limited and immune control models of HIV infection: a comparison. J. Theoret. Biol. 190 (1998) 201-214.

[7] Y. Dong, G. Huang, R. Miyazaki and Y. Takeuchi, Dynamics in a tumor immune system with time delays. Appl. Math. Comput. 252 (2015) 99-113.

[8] A.M. Elaiw and N.H. AlShamrani, Global stability of humoral immunity virus dynamics models with nonlinear infection rate and removal. Nonlinear Anal. Real World Appl. 26 (2015) 161-190.

[9] J.K. Hale and S.M.V. Lunel, Introduction to Functional Differential Equations. Springer Verlag, New York (1993).

[10] B.D. Hassard, N.D. Kazarionoff and Y.H. Wan, Vol. 41 of Theory and Applications of Hopf Bifurcation. CUP Archive (1981).

[11] Z. Hu, J. Liu, H. Wang and W. Ma, Analysis of the dynamics of a delayed HIV pathogenesis model. J. Math. Anal. Appl. 234 (2010) 461-476.

[12] Z. Hu, J. Zhang, H. Wang, W. Ma and F Liao, Dynamics of a delayed viral infection model with logistic growth and immune impairment. Appl. Math. Model. 38 (2014) 524-534.

[13] X. Lai and X. Zou, Modeling HIV-1 virus dynamics with both virus-to-cell infection and cell-to-cell transmission. SIAM J. Appl. Math. 74 (2014) 898-917.

[14] X. Lai and X. Zou, Modeling cell-to-cell spread of HIV-1 with logistic target cell growth. J. Math. Anal. Appl. 426 (2015) $563-584$.

[15] F. Li and J. Wang, Analysis of an HIV infection model with logistic target-cell growth and cell-to-cell transmission. Chaos Soliton Fract. 81 (2015) 136-145.

[16] X. Li and J. Wei, On the zeros of a fourth degree exponential polynomial with applications to a neural network model with delays. Chaos Solitons Fract. 26 (2005) 519-526.

[17] J.Z. Lin, R. Xu and X.H. Tian, Threshold dynamics of an HIV-1 virus model with both virus-to-cell and cell-to-cell transmissions, intracellular delay, and humoral immunity. Appl. Math. Comput. 315 (2017) 516-530.

[18] A. Murase, T. Sasaki and T. Kajiwara, Stability analysis of pathogen-immune interaction dynamics. J. Math. Biol. 51 (2005) $247-267$.

[19] Y. Muroya, Y. Enastu and H. Li, Global stability of a delayed HTLV-1 infection model with a class of non-linear incidence rates and CTL simmune response. Appl. Math. Comput. 219 (2013) 59-73.

[20] K. Pawelek, S. Liu, F. Pahlevani and L. Rong, A model of HIV-1 infection with two time delays: mathematical analysis and comparison with patient data. Math. Biosci. 235 (2012) 98-109.

[21] X.Y. Song, S.L. Wang and J. Dong, Stability properties and Hopf bifurcation of a delayed viral infection model with lytic immune response. J. Math. Anal. Appl. 373 (2011) 345-355.

[22] S. Tipsri and W. Chinviriyasit, The effect of time delay on the dynamics of an SEIR model with nonlinear incidence. Chaos Solitons Fract. 75 (2015) 153-172.

[23] C. vargas-de-leon, Stability analysis of a model for HBV infection with cure of infected cells and intracellula delay. Appl. Math. Comput. 219 (2012) 389-398.

[24] T. Wang, Z. Hu, F. Liao and W. Ma, Global stability analysis for delayed virus infection model with general incidence rate and humoral immunity. Math. Comput. Simulat. 89 (2013) 13-22.

[25] T.L. Wang, Z.X. Hu and F.C. Liao, Stability and Hopf bifurcation for a virus infection model with delayed humoral immunity response. J. Math. Anal. Appl. 411 (2014) 63-74.

[26] J. Wang, J. Lang and X. Zou, Analysis of an age structured HIV infection model with virus-to-cell infection and cell-to-cell transmission. Nonlinear Anal. Real World Appl. 34 (2017) 75-96.

[27] K.F. Wang, W.D. Wang, H.Y. Pang and X.N. Liu, Complex dynamic behavior in a viral model with delaye dimmune response. Physica D 226 (2007) 197-208

[28] K.F. Wang, W.D. Wang and X.N. Liu, Global stability in a viral infection model with lytic and nonlytic immune responses. Comput. Math. Appl. 51 (2006) 1593-1610.

[29] S. Wang and D. Zou, Global stability of in-host viral models with humoral immunity and intracellular delays. Appl. Math. Model. 36 (2012) 1313-1322. 